\title{
Comparative studies of mucosal humoral and cellular immune responses to 2009 pandemic $\mathrm{H} 1 \mathrm{~N} 1$ influenza virus in mice
}

\author{
Y.-Y. ZHANG ${ }^{1 *}$, J. AN ${ }^{1 *}$, K. WANG ${ }^{1 *}$, B. HAN ${ }^{1}$, Y.-F. LIU' ${ }^{1}$, H.-L. JIA ${ }^{1}$, Y.-S. LI², L. LEI ${ }^{1}$, N. JIA ${ }^{3 *}$, J. LV ${ }^{1^{*}}$ \\ ${ }^{1}$ The General Hospital of the PLA Rocket Force, Beijing, 100088, P. R. China; ${ }^{2}$ Chinese PLA General Hospital, Beijing, 10853 , \\ P. R. China; ${ }^{3}$ State Key Laboratory of Pathogen and Biosecurity, Beijing Institute of Microbiology and Epidemiology, \\ Beijing, 100071, P. R. China
}

Received June 11, 2018; revised September 14, 2018; accepted May 10, 2019

\begin{abstract}
Summary. - The nasal-associated lymphoid tissues (NALTs), embedded in the submucosa of murine upper respiratory tract, represents an important site of induction for local mucosal immune responses to airborne pathogens and intranasal vaccines. Here, we systematically investigated the mucosal humoral and cellular immune responses of NALTs in mice infected with A/Beijing/501/2009 (BJ501) and A/Puerto Rico/8/1934 (PR8) viruses. Compared with PR8 infection, BJ501 induced a more rapid increase of virus-specific IgA and IgG antibodies in the nasal lavage fluid and a higher ratio of $\operatorname{IgG} 1 / \operatorname{IgG} 2 \mathrm{a}$, indicating a stronger Th2 response to BJ501 in mucosal immunity. In addition, using virus-specific enzyme-linked immunospot assay (ELISpot assay), we observed higher and earlier responses of virus-specific IgA and IgG antibody-secreting cells (ASCs) and IFN- $\gamma$ and IL- 4 cytokine-secreting cells (CSCs) in NALTs of mice intranasally infected with BJ501 virus. In particular, the frequency of BJ501-specific IFN- $\gamma$-CSCs significantly correlated with the kinetics of BJ501 virus load in NALTs, suggesting an important role of IFN- $\gamma$-CSCs-associated mucosal cellular immune responses in BJ501 virus clearance. Collectively, BJ501 induced a more comprehensive and rapid mucosal immune responses in NALTs of mice, providing further understanding of the immune responses elicited by 2009 pandemic H1N1 virus in upper respiratory tract.
\end{abstract}

Keywords: nasal-associated lymphoid tissues (NALTs); influenza virus; mucosal immune response; Th1/Th2 response

\section{Introduction}

Influenza is a highly acute respiratory disease caused by influenza virus infection of the upper and lower respiratory tracts of the host and poses a huge threat to the worldwide

*Corresponding authors. E-mails: lvjin6630@hotmail.com (J. Lv), jiana79_41@hotmail.com (N. Jia); phone: +86-10-66343284, 8610-66948442. "Three authors contributed equally.

Abbreviations: ASC $=$ antibody-secreting cell; BJ501 = A/Beijing/501/2009; CSC = cytokine-secreting cell; ELISpot = enzymelinked immunospot; IFN- $\gamma=$ interferon gamma; $\operatorname{Ig}(A, G, M)=$ immunoglobulin (A,G,M); IL-4 = interleukin 4; NALT(s) = nasalassociated lymphoid tissue(s); PR8 = A/Puerto Rico/8/1934; SFU $=$ spot forming unit; Th $(1,2)=$ T Helper $(1,2)$; URT $=$ upper respiratory tract public health (Stohr, 2002). In 2009, at least 284,000 people died in the first 12 months of the pandemic caused by the highly contagious novel H1N1 influenza virus strain (Dawood et al., 2012). Given that the respiratory tract is the first site exposed to inhaled influenza virus invasion and is equipped with a network of lymphoid tissues, the mucosal immune responses to 2009 pandemic H1N1 influenza virus may be associated with the host immune response and affect the severity of the respiratory disease (Ma et al., 2011). Our previous study demonstrated that, in response to lower respiratory tract (LRT) infection, the 2009 pandemic $\mathrm{H} 1 \mathrm{~N} 1$ influenza virus strain A/Beijing/501/2009 (BJ501, H1N1) caused different pulmonary immunity and fatal illness patterns in mice compared with the mouse-adapted pathogenic influenza virus strain A/Puerto Rico/8/1934 (PR8, H1N1) (Lv et al., 2014). However, the responses of mucosal humoral 
and cellular immunities in the upper respiratory tract (URT) following 2009 pandemic H1N1 influenza virus infection are less well understood.

In the upper respiratory tract of mice, highly organized lymphoid tissues located at the base of the nasal cavities, named nasal-associated lymphoid tissues (NALTs), have been widely regarded as the functional equivalent of the Waldeyer's ring of humans and Peyer's patches of the gut (Kiyono and Fukuyama, 2004; Randall and Mebius, 2014). NALTs are known to be involved in the induction of local mucosal as well as systemic immunity after intranasal administration of antigens or viral vaccines (Hoft et al., 2017; Yang et al., 2011). Antiviral IgA and IgG antibodies in the upper respiratory tract were considered to be the primary mechanisms of mucosal immunity against PR8 influenza virus infection (Tamura et al., 1998; Watanabe et al., 2002). Recently, NALTs were also found to be an induction site for recalling memory CD8+ T cells after PR8 influenza virus infection, and played an important role in cytotoxic T lymphocyte (CTL) immunity against pathogenic influenza virus (Pizzolla et al., 2017). Nevertheless, the participation of the local mucosa-associated lymphoid tissues in generation of protective immunity against 2009 pandemic BJ501 influenza strain infection remains to be elucidated.

In the present study, we systematically assessed the in vivo kinetics of viral-specific serum antibodies against BJ501 and PR8 influenza virus strains and compared the mucosal humoral and cellular immune responses of different influenza strains in mice.

\section{Materials and Methods}

Ethics statement. The animal research was approved by the Animal Subjects Research Review Board of the Beijing Institute of Microbiology and Epidemiology (approval No. SYKX-2007-005), and was carried out in strict accordance with the recommendations in the Guide for Care and Use of Laboratory Animals of the National Institutes of Health.

Mice. Female BALB/c mice at 6-8-week-old were purchased from the Institute of Jingfeng Medical Laboratory Animal and housed in the Animal Care Facilities under BSL2 pathogen-free conditions. Accordingly, all possible care was taken to minimize suffering.

Viruses. The mouse-adapted pathogenic A/Puerto Rico/8/1934 (H1N1) influenza virus strain was kindly provided by Dr. Yue-long Shu, Chinese Center for Disease Control and Prevention. The influenza H1N1 virus A/Beijing/501/2009 (BJ501) was isolated from a patient from Beijing in 2009 and preserved in our lab. Viruses were propagated by inoculation into 10-day-old specific-pathogen free (SPF) chicken embryos (Laboratory Animal Center, Beijing, China) via the allantoic route. Infected allantoic fluids were pooled and stored at $-80^{\circ} \mathrm{C}$ until use. The $50 \%$ tissue culture infectious dose $\left(\mathrm{TCID}_{50}\right)$ for each virus was determined by serial dilution of the virus in Madin-Darby canine kidney (MDCK) cells (ATCC, Virginia, USA) and calculated by the previously described method (Neumann and Kawaoka, 2001). The viral titers were determined by standard plaque assays in MDCK cells as previously described (Zeng et al., 2007). After $48 \mathrm{~h}$ of incubation at $37^{\circ} \mathrm{C}$, plaques were stained with $0.1 \%$ crystal violet and counted. All experiments with live influenza viruses were performed in the approved bio-safety level 3 animal facilities.

Viral infection of mice. Viral infections of BJ501 and PR8 in mice were performed as described previously (Lv et al., 2014). Briefly, a total of 105 mice in each group were anesthetized with inhalation diethyl ether and intranasally inoculated with $25 \mu$ of virus suspension containing $5 \times 10^{5} \mathrm{PFU}$ of BJ501 or PR8 virus in phosphate buffered saline (PBS). In addition, a normal control group was given intranasal PBS (mock treatment). Mice were sacrificed at different time points $(2,4,6,8,10,12,14$ days) after virus infection, and the nasal lavage fluid and nasal-associated lymphoid tissues (NALTs), respectively, were collected from 15 mice in each group.

Preparation of nasal lavage fluid. For nasal lavage recovery, the nasal cavity was stripped out and washed with three separate injections of $0.2 \mathrm{ml}$ sterile PBS at room temperature through a $200 \mu \mathrm{l}$ pipette. The collected nasal lavage fluid was centrifuged at $1000 \times \mathrm{g}$ for $15 \mathrm{~min}$. The supernatants were recovered and stored at $-20^{\circ} \mathrm{C}$ for antibody titer analysis by ELISA.

Preparation of NALT lymphocytes. Mice were sacrificed on days $2,4,6,8,10,12$ and 14 after influenza virus infection, and their NALT lymphocytes were separated from infected mice, as described previously (Tamura et al., 1998). In brief, the NALTs, localized bilaterally on the posterior side of the palate, were removed from the nasal cavity by cutting along the inside edges of the upper molars with a scalpel and then peeling the tissue away from the roof of the mouth. The NALT fragments were mechanically disrupted through a stainless-steel mesh and NALT lymphocytes were released in $5 \mathrm{ml}$ complete RPMI 1640 medium containing 2\% fetal calf serum (FCS). Viable lymphocytes were further purified by red blood cell lysing buffer ( $0.16 \mathrm{M} \mathrm{NH}_{4} \mathrm{Cl}, 0.17 \mathrm{M}$ Tris- $\left.\mathrm{HCl}, \mathrm{pH} 7.4\right)$ and isolated by centrifugation at $1000 \times \mathrm{g}$ for $10 \mathrm{~min}$.

Quantitative analysis of viral RNA. Total RNA was extracted from the fresh NALTs using Trizol (Invitrogen, USA) reagent according to the manufacturer's instructions. For viral load quantification, a single-tube, real-time Taqman RT-PCR assay was performed using the Taqman one-step RT-PCR Master Mix in the Roche Light Cycler 480 II Real-Time PCR Detection System (Roche Ltd., Switzerland). The primers and probe were designed according to the conserved region of the influenza matrix (M) gene for PR8 as previously described (Jelley-Gibbs et al., 2007), and for the detection of BJ501, the primers and probe were designed according to the Centers for Disease Control and Prevention (CDC) protocol for swine influenza A virus (Bolotin et al., 2009).

ELISpot (enzyme-linked immunospot) assay for antibody secreting cells (ASCs) and cytokine secreting cells (CSCs). NALT lymphocytes were obtained from mice after infection at different time points, and 


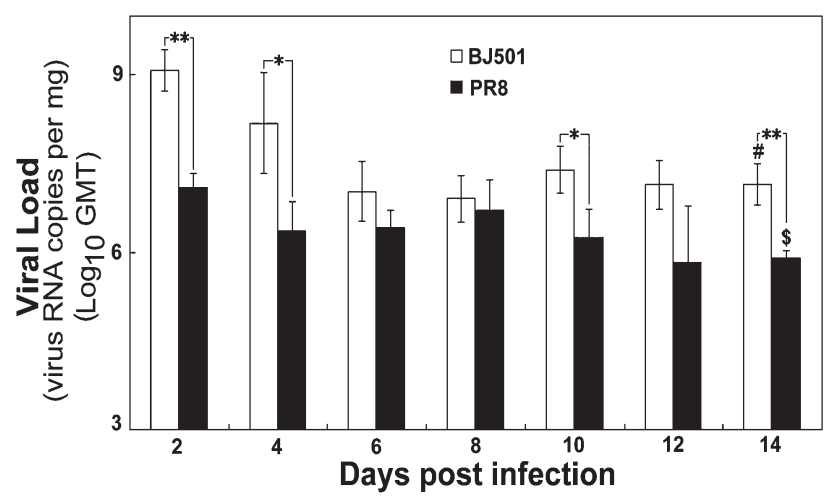

Fig. 1

Viral load of BJ501 and PR8 viruses in NALTs of mice Viral loads were determined in fresh NALTs at each time point postinfection by real time RT-PCR analysis and were represented as viral RNA copies per milligram NALT tissue. Mean viral load is representative of 5-6 mice NALTs per time point/group, and the bars indicate the standard deviations. ${ }^{\$} \mathrm{p}<0.05$, compared among different time points within BJ501 virus infection, " compared among different time-points within PR8 virus infection, ${ }^{*} p<0.05$ and ${ }^{* *} p<0.01$, compared between BJ501 and PR8 infections at the same time point.

the single-lymphocyte suspensions were prepared for quantifying virus-specific ASCs and CSCs using the ELISpot 96-well plates (Millitier HA; Millipore). According to the purpose, the ELISpot plate was coated with $2.5 \mu \mathrm{g} / \mathrm{ml}$ purified PR8 or BJ501 antigen for influenza virus-specific ASCs determination, or coated with anti-mouse IFN- $\gamma$, and IL- 4 capture antibody for IFN- $\gamma$ - and IL-4-producing cells detection. A total of $2 \times 10^{5}$ cells of lymphocytes suspensions were added to each well. All ELISpot assays were performed according to our previously described procedure (Lv et al., 2014).

Measurement of antibody level by enzyme-linked immunosorbent assay (ELISA). ELISA was carried out as described previously in plates coated with $1 \mu \mathrm{g} / \mathrm{ml}$ per well of inactivated PR8 or BJ501 influenza virus (Lv et al., 2014). For the detection of influenza-specific mucosal antibodies, the goat anti-mouse $\operatorname{IgA}, \operatorname{IgM}$ (Invitrogen), goat anti-mouse IgG (Jackson ImmunoResearch Laboratories) and goat anti-mouse IgG1, IgG2b, IgG2a, IgG2c, and IgG3 (Bethyl laboratories, Inc.) antibodies conjugated with horseradish peroxidase were used as secondary antibodies. The virus-specific antibody titer was defined as the reciprocal value of the highest sample dilution giving an absorbance value greater than twice that of the samples from the negative controls. The titers were gained in duplicate.

Statistical analysis. Statistical differences at each time point within one group were determined by one-way ANOVA test, while statistical differences of the two viruses at the same time point were determined by independent-sample t-test with SPSS19.0 software (Chicago, IL, USA). The F-statistics and t-statistics, which indicated the significant levels of statistics test, were determined as the ratios of variation between sample means and variation within the samples. Values of $p<0.05$ were considered significant.

\section{Results}

The kinetics of viral RNA expression in NALTs of mice infected with BJ501 and PR8

To elucidate the mucosal immune responses of URT after influenza virus infection, the kinetics of viral replication in NALTs of mice was firstly examined following intranasal infection with the same dose of BJ501 and PR8 viruses. Although replicating well in vivo, both viruses exhibited significantly different viral replication kinetics in the NALTs of mice. BJ501 had a significant decrease on day 6 after infection before replicating at a stable level $(\mathrm{F}=7.85$, $p=0.001, p<0.01$ ), whereas PR8 virus maintained a relatively stable replication level throughout the observation period. However, the replication of BJ501 virus in the NALTs of mice was generally higher than that of the PR8 virus, with significant discrepancies on days $2,4,10$ and $14(\mathrm{t}=8.08$, $p=0.001 ; \mathrm{t}=3.24, p=0.032 ; \mathrm{t}=3.19, p=0.033$ and $\mathrm{t}=6.11$, $p=0.004)$ (Fig. 1).

\section{Virus-specific nasal antibody responses in mice infected} with BJ501 and PR8

The virus-specific antibodies, including $\operatorname{IgA}, \operatorname{Ig} M$ and IgG isotypes, were examined in the nasal lavage fluid of mice infected with BJ501 or PR8 virus. BJ501-specific IgA and IgG antibodies in nasal wash increased early, from day 2 post-infection, rose to their highest level on day 8 and remained stable until day $14(\mathrm{~F}=20.88, p=0.000$ for $\operatorname{IgA}$ and $\mathrm{F}=77.09, p=0.000$ for IgG) (Fig. 2a). Unlike antibodies to $\mathrm{BJ} 501$ virus, anti-PR8 virus IgA and IgG antibodies were first notably detected in the nasal wash on day 8 , increased to peak on day 10 , and maintained a significant level through day $14(\mathrm{~F}=52.54, p=0.000$ for $\operatorname{IgA}$ and $\mathrm{F}=131.69, p=0.000$ for $\operatorname{IgG})$, although the titer of $\operatorname{IgA}$ antibodies was slightly decreased on day 12 (Fig. 2a). However, the IgM responses, which were considered to be $\mathrm{T}$ cell-independent, were induced in nasal lavage fluid of mice infected with BJ501 and $\mathrm{PR} 8$ viruses $(\mathrm{F}=40.29, p=0.000$ for $\mathrm{BJ} 501$ and $\mathrm{F}=49.73$, $p=0.000$ for PR8) on day 2 and exhibited no significant differences in titer kinetics during the study (Fig. 2a).

We further compared the profiles of specific IgG isotypes response to BJ501 virus and PR8 virus from day 8 to day 14 after viral infection. As shown in Figure $2 b$, the viruses induced four subtypes of IgG antibodies in the nasal lavage fluid, including IgG1, IgG2a, IgG2b and IgG3. The dynamic response pattern of IgG3 antibody was similar to that of IgG1, and IgG2a was similar to IgG2b. No detectable IgG2c was found in this study (data not shown). However, the ratio of IgG1 to IgG2a, an indicator of Th2 response, increased gradually to its maximum level on day 8 , was significantly higher in BJ501-infected mice than in PR8-infected mice 
(a)

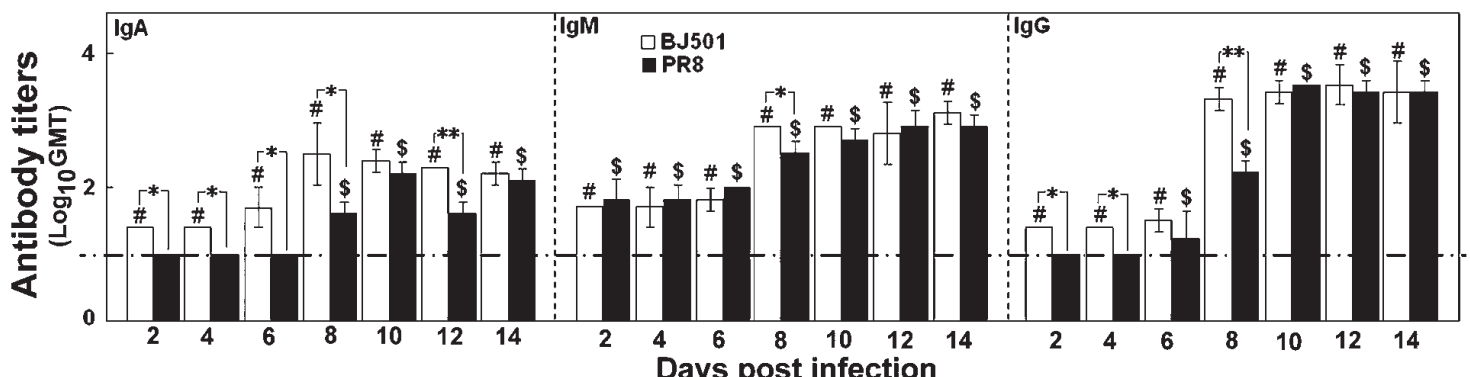

(b)

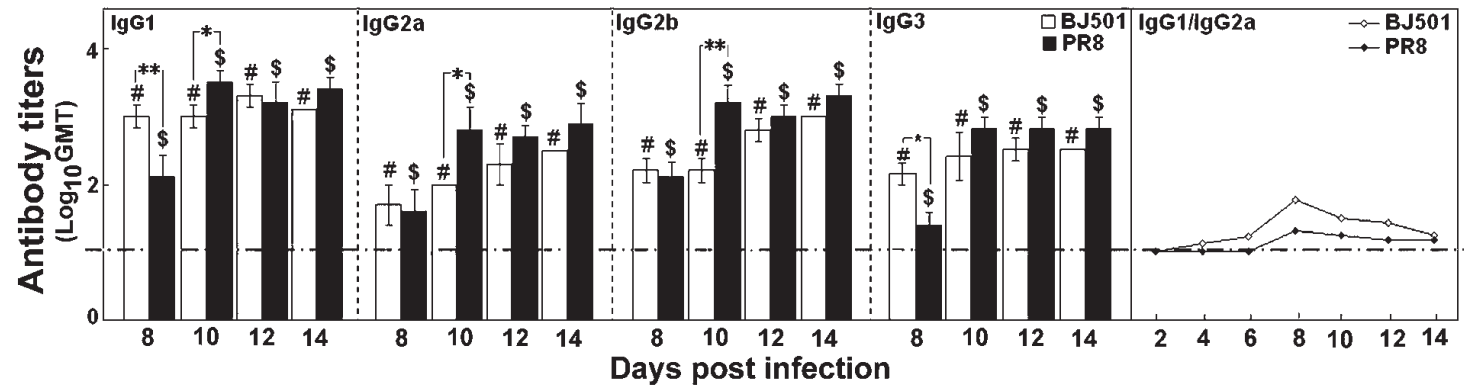

Fig. 2

Virus-specific nasal antibody responses in the NALTs of mice with influenza virus infection

Titers of virus-specific IgA, IgM, IgG antibodies (a) and specific IgG isotypes including IgG1, IgG2a, IgG2b and IgG3 antibodies (b) in the NALTs of mice after infection with $5 \times 10^{5}$ PFU of BJ501 or PR8 virus. Five mice in each group were sacrificed at each time point post infection, and virus-specific antibodies in NALTs were determined by ELISA. Mice without infection were used as the control group. The dotted lines depict the control level. ${ }^{\$} p<0.05$, compared among different time points within BJ501 virus infection, ${ }^{*}$ compared among different time points within PR8 virus infection, ${ }^{*} p<0.05$ and ${ }^{* *} p<0.01$, compared between BJ501 and PR8 infections at the same time point.

$(\mathrm{t}=3.60, p=0.022)$ during the study; thus it suggested a stronger Th2 response predominance in the BJ501 infection.

Virus-specific ASCs responses in NALTs of mice infected with BJ501 and PR8

Owing to the importance of ASCs in nasal antibody production and maintenance, we further assessed the frequencies and Ig isotype pattern (IgA and IgG) of virus-specific ASCs responses in the NALTs of mice infected intranasally with BJ501 and PR8 (Fig. 3). The peak of BJ501-specific $\mathrm{IgA}^{+}$ASCs responses was first detected on day 8 and dramatically declined thereafter to approximate the baseline on day $14(\mathrm{~F}=466.54, p=0.000)$ (Fig. 3a). The frequency of PR8-specific IgA $\mathrm{A}^{+}$ASCs, peaked on day 10 , was about five times lower than the peak frequency of BJ501 specific $\mathrm{IgA}^{+}$ASCs, and dropped sharply to the baseline on day 12 $(\mathrm{F}=33.84, p=0.000)$ (Fig. 3a). In contrast, the responses of virus-specific $\operatorname{IgG}^{+}$ASCs in NALTs exhibited an earlier and more robust pattern than IgA ${ }^{+}$ASCs. Both BJ501 and PR8 virus-specific IgG ${ }^{+}$ASCs appeared first on day 6 after infection, and BJ501 reached its peak on day 8, earlier than that of PR8 on day $10(\mathrm{~F}=188.50, p=0.000$ for BJ501 and $\mathrm{F}=96.05, p=0.000$ for PR8) (Fig. 3b). Moreover, the frequency of BJ501-specific IgG ${ }^{+}$ASCs was significantly higher than that of PR8 $(p<0.05$, and $p<0.01)$ in the course of study
(Fig. 3b). These data suggested that BJ501 induced a stronger and faster mucosal humoral immune responses than PR8 in in NALTs of mice.

Virus-specific CSCs responses in NALTs of mice infected with BJ501 and PR8

The frequencies of IFN- $\gamma$ secreting cells (IFN- $\gamma$-CSCs) and IL-4 secreting cells (IL4-CSCs) are generally considered to be the indicators of Th1 dominance and Th2 dominance in the cellular immune response, respectively. As shown in Figure 4, BJ501-specific IFN- $\gamma$-CSCs responses were first detected in NALTs of mice on day 4, peaked on day 6, and slowly decreased to $1 / 5$ of the peak on day 12 . Interestingly, the frequency of BJ501-specific IFN- $\gamma$-CSCs had a slight recovery on day $14(\mathrm{~F}=107.62, p=0.000)$ (Fig. 4a). In contrast, the PR8-specific IFN- $\gamma$-CSCs responses, significantly lower than BJ501-specific $(p<0.01)$, were first detected to peak on day 6 and then significantly dropped to the baseline on day 8 and day $14(\mathrm{~F}=49.22, p=0.000)$ (Fig. 4a). For Th2 responses, the frequencies of IL-4-CSCs in BJ501-infected NALTs were significantly higher than those in PR8-infected NALTs during the study $(p<0.05$ and $p<0.01)$. Moreover, the IL-4-CSCs were first detected on day 6 in BJ501-infected NALTs, earlier than in PR8-infected on day 10 (Fig. 4b). These results suggested that BJ501 induced a stronger mu- 


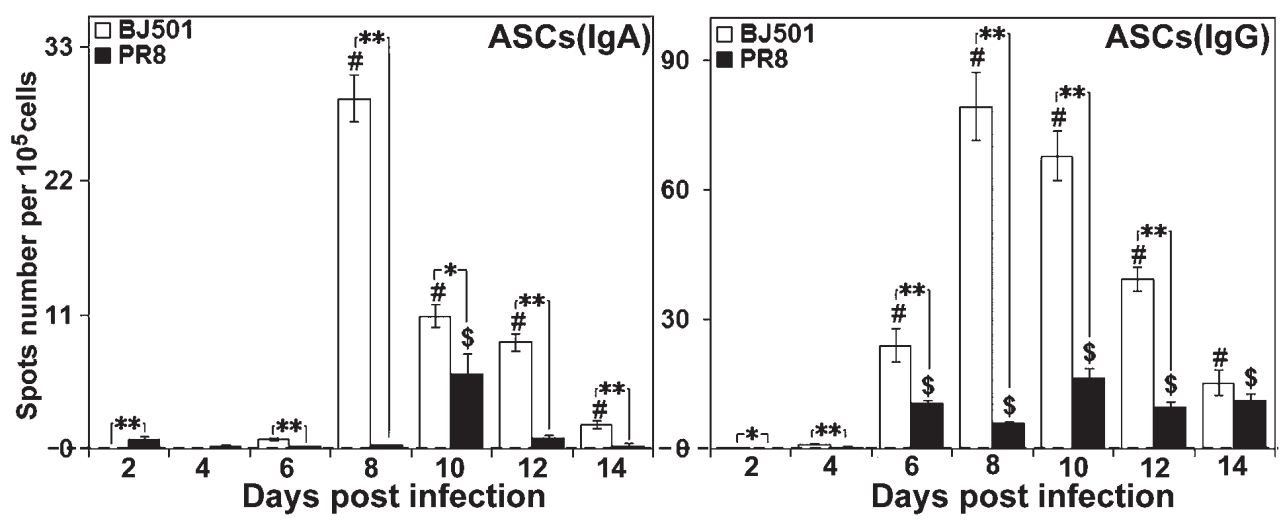

Fig. 3

Influenza virus-specific ASCs responses in NALTs of mice infected with BJ501 or PR8

The frequencies of influenza virus-specific IgA (a) and IgG (b) ASCs (mean SFU/10 5 cells) in NALTs of five mice in each group were determined by ELISpot analyses at different time points after infection with $5 \times 10^{5} \mathrm{PFU}$ of BJ501 or PR8 virus. The data are represented as mean SFU $/ 10^{5}$ cells \pm standard deviation. The dotted line at the bottom is the background of the PBS control. ${ }^{\$} p<0.05$, compared among different time points within BJ501 virus infection, ${ }^{*}$ compared among different time points within PR8 virus infection, ${ }^{\star} p<0.05$ and ${ }^{\star *} p<0.01$, compared between BJ501 and PR8 infection at the same time point.

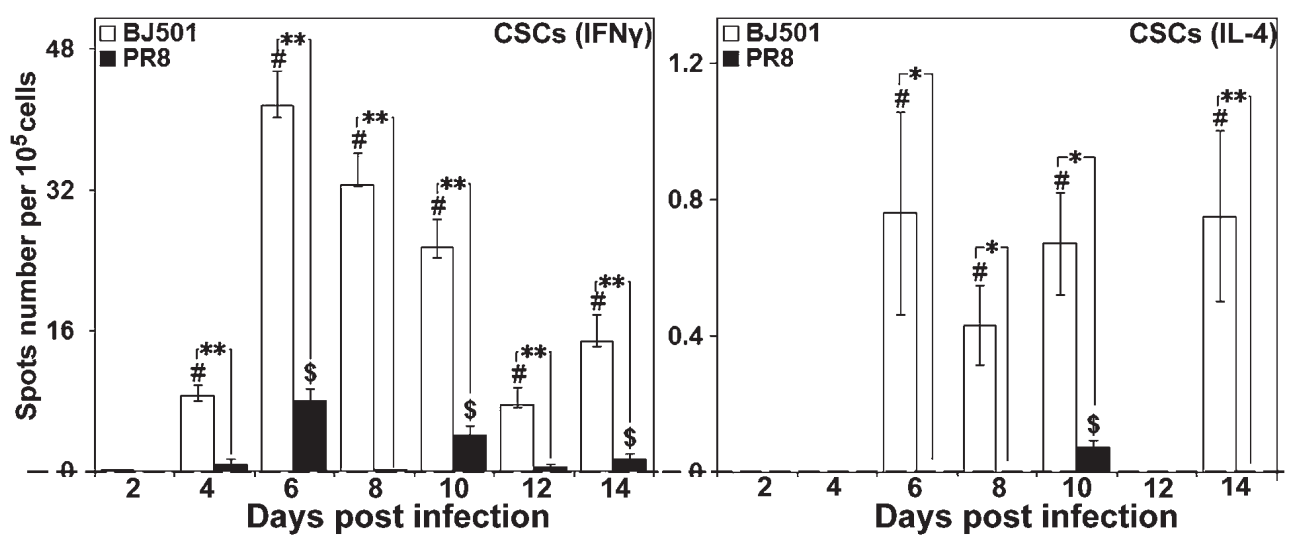

Fig. 4

Influenza virus-specific CSCs responses in NALTs of mice infected with BJ501 and PR8

The frequencies of influenza virus-specific IFN- $\gamma^{+}(\mathbf{a})$ and IL- $4^{+}(\mathbf{b})$ CSCs (mean SFU/10 $0^{5}$ cells) in NALTs of five mice in each group were determined by ELISpot analyses at different time points after infection with $5 \times 10^{5}$ PFU of BJ501 or PR8 viruses. The data are represented as mean SFU/10 5 cells \pm standard deviation. The dotted line at the bottom is the background of the PBS control. ${ }^{\$} p<0.05$, compared among different time points within BJ501 virus infection, ${ }^{*}$ compared among different time points within PR8 virus infection, ${ }^{*} p<0.05$ and ${ }^{* *} p<0.01$, compared between BJ501 and PR8 infection at the same time point.

cosal cellular immune response than PR8 in NALTs of mice, particularly the Th1 immune responses.

\section{Discussion}

The upper respiratory tract (URT), as well as the region where intranasal vaccines are inoculated, plays an important role as the first line of defense for human respiratory pathogens, including influenza virus (Rudraraju et al., 2014; Surman et al., 2014). The organized NALT, one of the most important primary lymphoid organs in URT of mice, has evolved to promote the induction of a strong local mucosal immune response to the respiratory pathogens (Petukhova et al., 2009; Wiley et al., 2005). Here, we systematically analyzed the mucosal humoral and cellular immune responses of NALTs in mice intranasally infected with BJ501 and PR8 influenza viruses. We discovered that the 2009 pandemic H1N1 influenza virus strain BJ501 induced a more comprehensive and rapid mucosal immune responses than PR8 in NALTs of mice.

The viral replication efficiency of different influenza virus strains causing different mucosal immune responses remains 
largely unknown. Our previous study demonstrated that the pulmonary viral load in PR8-infected lung was significantly higher than that in BJ501-infected, and the decrease of viral load was significantly earlier in the lungs of mice infected with BJ501 virus (from day 10 post infection) than in those infected with PR8 (from day 12 post infection) (Lv et al., 2014). Interestingly, we here found that unlike the case in the lung, BJ501 virus had a higher mucosal viral replication efficiency in NALTs and decreased on day 6 after infection, whereas PR8 virus maintained a relatively lower and stable viral replication. To our knowledge, the 2009 pandemic $\mathrm{H} 1 \mathrm{~N} 1$ virus is prone to respiratory transmission and slightly more pathogenic than the seasonal $\mathrm{H} 1 \mathrm{~N} 1$ viruses (Munster et al., 2009), probably because of the higher viral replication activity of BJ501 virus in URT observed in our experiments.

Previous clinical reports indicated that cytokine storm is involved in the pathology of severe 2009 pandemic influenza, and mucosal immunity in NALTs is the initial step leading to cytokine storm (Cheng et al., 2011; Yang et al., 2012). However, the underlying immune mechanism in NALTs is unclear. In the present study, we demonstrated that BJ501 induced a more rapid increase of virus-specific IgA and IgG antibodies in the nasal lavage fluid and a higher ratio of IgG1 to IgG2a isotype, an indicator of Th2 response in mucosal immunity. The Th2-biased response was considered to be helpful for the humoral immune maturation against extracellular pathogens by activation of antibody-secreting B lymphocytes (Mosmann et al., 2009; Peterson et al., 1998). IL-4 secretion was the representative of Th2 response. Indeed, we observed a significantly higher frequency of IL-4-CSCs induced by BJ501 infection in NALTs. Following exposure to the milieu of IL- 4 secretion and viral infection, the native B cells in the germinal center of NALTs are stimulated to enter into a process of clonal expansion and differentiate into virus-specific IgA and IgG ASCs. It had been demonstrated that ASCs generation from specific B cell precursors (Asanuma et al., 1998; Chiu et al., 2015; Tamura et al., 1998) was closely related to the production of mucosal antibodies. Thus, the rapid increase of BJ501-specific IgA and IgG antibodies in nasal washes is probably caused by the fact that BJ501 induced a more rapid activation of virus-specific ASCs than PR8 (peaked at day-8vs. at day-10). In addition, the frequencies of virus-specific IgA and IgG ASCs in BJ501infected NALTs were also significantly higher than those in PR8-infected NALTs, indicating that faster and more pronounced mucosal humoral immune responses were induced by intranasal BJ501 virus infection.

Previous studies indicated that IgA antibodies in the nasal lavage fluid played a key role in virus clearance during PR8 infection in mice (Sakuma et al., 2015; Tamura et al., 1998). However, under our current experimental conditions, we failed to observe a significant correlation between mucosal IgA antibody levels and the virus clearance in the process of BJ501 infection, with the evidence that the viral load of BJ501 in NALTs had a sharp decrease on day 6 after infection, while the BJ501-specific IgA antibody in nasal lavage fluid increased to peak levels on day 8 after infection. That might be due to the larger volume $(25 \mu \mathrm{l} v s .2 \mu \mathrm{l})$ of influenza virus used for intranasal inoculation in the present study, where we established an infection model that localized the virus to total respiratory tract (Lv et al., 2014), rather than to the upper airways as in the previous studies (Asanuma et al., 1998; Sakuma et al., 2015; Tamura et al., 1998). Interestingly, we found that intranasal BJ501 virus infection could induce a pronounced virus-specific IFN- $\gamma$-CSCs response in NALTs, which is considered the representative of Th1 response against intracellular pathogens by activation of cytotoxic T cells and NK cells (Miller et al., 2009; Yang et al., 2017). Moreover, BJ501-specific IFN- $\gamma$-CSCs responses were first detected in NALTs of mice on day 4 and peaked on day 6 , which significantly correlated with the kinetics of BJ501 virus load in NALTs. The present observation that BJ501 virus clearance in NALTs seemsto be mediated mainly by IFN- $\gamma$-CSCs response indicates that mucosal cellular immune responses against influenza might be more important than mucosal IgA or IgG antibody for hindering the spread of 2009 pandemic H1N1 virus in the population.

In summary, we demonstrated that following intranasal virus infection both BJ501 and PR8 could replicate efficiently and induce pronounced mucosal humoral and cellular immune responses in NALTs of mice. Specifically, the 2009 pandemic $\mathrm{H} 1 \mathrm{~N} 1$ virus strain BJ501 induced a more comprehensive and rapid mucosal immune responses than PR8 in NALTs of mice, and mucosal IFN- $\gamma$-CSCs responses served as the primary mechanism for BJ501 virus clearance in NALTs. These results have important implications for better understanding of the immune response initiated following 2009 pandemic H1N1 virus infection in the URT.

Acknowledgments. The authors thank Dr. Yue-long Shu (Chinese National Influenza Center), for providing experimental virus strains, and Mr. Shi-jia Pei (Cellular Technology Ltd.) for ELISpot reading. This study was supported by the State Key Research Development Program of China (2016YFC 1200301).

\section{References}

Asanuma H, Aizawa C, Kurata T, Tamura S (1998): IgA antibodyforming cell responses in the nasal-associated lymphoid tissue of mice vaccinated by intranasal, intravenous and/ or subcutaneous administration. Vaccine 16, 1257-1262. https://doi.org/10.1016/S0264-410X(98)00048-6

Bolotin S, Robertson AV, Eshaghi A, De Lima C, Lombos E, ChongKing E, Burton L, Mazzulli T, Drews SJ (2009): Development of a novel real-time reverse-transcriptase PCR method for the detection of $\mathrm{H} 275 \mathrm{Y}$ positive influenza A 
H1N1 isolates. J. Virol. Methods 158, 190-194. https:// doi.org/10.1016/j.jviromet.2009.01.016

Cheng XW, Lu J, Wu CL, Yi LN, Xie X, Shi XD, Fang SS, Zan H, Kung HF, He ML (2011): Three fatal cases of pandemic 2009 influenza A virus infection in Shenzhen are associated with cytokine storm. Respir. Physiol. Neurobiol. 175, 185-187. https://doi.org/10.1016/j.resp.2010.11.004

Chiu C, Ellebedy AH, Wrammert J, Ahmed R (2015): B cell responses to influenza infection and vaccination. Curr. Top. Microbiol. Immunol. 386, 381-398. https://doi. org/10.1007/82 $2014 \quad 425$

Dawood FS, Iuliano AD, Reed C, Meltzer MI, Shay DK, Cheng PY, Bandaranayake D, Breiman RF, Brooks WA, Buchy P, Feikin DR, Fowler KB, Gordon A, Hien NT, Horby P, Huang QS, Katz MA, Krishnan A, Lal R, Montgomery JM, Molbak K, Pebody R, Presanis AM, Razuri H, Steens A, Tinoco YO, Wallinga J, Yu H, Vong S, Bresee J, Widdowson MA (2012): Estimated global mortality associated with the first 12 months of 2009 pandemic influenza A H1N1 virus circulation: a modelling study. Lancet Infect. Dis. 12, 687-695. https://doi.org/10.1016/ $\underline{\text { S1473-3099(12)70121-4 }}$

Hoft DF, Lottenbach KR, Blazevic A, Turan A, Blevins TP, Pacatte TP, Yu Y, Mitchell MC, Hoft SG, Belshe RB (2017): Comparisons of the Humoral and Cellular Immune Responses Induced by Live Attenuated Influenza Vaccine and Inactivated Influenza Vaccine in Adults. Clin. Vaccine Immunol. 24. pii: e00414-16. https://doi.org/10.1128/ CVI.00414-16

Jelley-Gibbs DM, Dibble JP, Brown DM, Strutt TM, McKinstry KK, Swain SL (2007): Persistent depots of influenza antigen fail to induce a cytotoxic CD8 $\mathrm{T}$ cell response. J. Immunol. 178, 7563-7570. https://doi.org/10.4049/ jimmunol.178.12.7563

Kiyono H, Fukuyama S (2004): NALT- versus Peyer's-patch-mediated mucosal immunity. Nat. Rev. Immunol. 4, 699-710. https://doi.org/10.1038/nri1439

Lv J, Wang D, Hua YH, Pei SJ, Wang J, Hu WW, Wang XL, Jia N, Jiang QS (2014): Pulmonary immune responses to 2009 pandemic influenza A (H1N1) virus in mice. BMC Infect. Dis. 14, 197. https://doi.org/10.1186/1471-2334-14-197

Ma W, Belisle SE, Mosier D, Li X, Stigger-Rosser E, Liu Q, Qiao C, Elder J, Webby R, Katze MG, Richt JA (2011): 2009 pandemic H1N1 influenza virus causes disease and upregulation of genes related to inflammatory and immune responses, cell death, and lipid metabolism in pigs. J. Virol. 85, 11626-11637. https://doi.org/10.1128/ IVI.05705-11

Miller CM, Smith NC, Ikin RJ, Boulter NR, Dalton JP, Donnelly $S$ (2009): Immunological interactions between 2 common pathogens, Th1-inducing protozoan Toxoplasma gondii and the Th2-inducing helminth Fasciola hepatica. PLoS One 4, e5692. https://doi.org/10.1371/journal. pone. 0005692

Mosmann TR, Kobie JJ, Lee FE, Quataert SA (2009): T helper cytokine patterns: defined subsets, random expression, and external modulation. Immunol. Res. 45, 173-184. https://doi.org/10.1007/s12026-009-8098-5
Munster VJ, de Wit E, van den Brand JM, Herfst S, Schrauwen EJ, Bestebroer TM, van de Vijver D, Boucher CA, Koopmans M, Rimmelzwaan GF, Kuiken T, Osterhaus AD, Fouchier RA (2009): Pathogenesis and transmission of swine-origin $2009 \mathrm{~A}(\mathrm{H} 1 \mathrm{~N} 1)$ influenza virus in ferrets. Science 325, 481-483. https://doi.org/10.1126/science. 1177127

Neumann G, Kawaoka Y (2001): Reverse genetics of influenza virus. Virology 287, 243-250. https://doi.org/10.1006/ viro.2001.1008

Peterson JD, Herzenberg LA, Vasquez K, Waltenbaugh C (1998): Glutathione levels in antigen-presenting cells modulate Th1 versus Th2 response patterns. Proc. Natl. Acad. Sci. USA 95, 3071-3076. https://doi.org/10.1073/ pnas.95.6.3071

Petukhova G, Naikhin A, Chirkova T, Donina S, Korenkov D, Rudenko L (2009): Comparative studies of local antibody and cellular immune responses to influenza infection and vaccination with live attenuated reassortant influenza vaccine (LAIV) utilizing a mouse nasalassociated lymphoid tissue (NALT) separation method. Vaccine 27, 2580-2587. https://doi.org/10.1016/j.vaccine.2009.02.035

Pizzolla A, Wang Z, Groom JR, Kedzierska K, Brooks AG, Reading PC, Wakim LM (2017): Nasal-associated lymphoid tissues (NALTs) support the recall but not priming of influenza virus-specific cytotoxic T cells. Proc. Natl. Acad. Sci. USA 114, 5225-5230. https://doi.org/10.1073/ pnas. 1620194114

Randall TD, Mebius RE (2014): The development and function of mucosal lymphoid tissues: a balancing act with microorganisms. Mucosal Immunol 7, 455-466. https://doi. org/10.1038/mi.2014.11

Rudraraju R, Jones BG, Surman SL, Sealy RE, Thomas PG, Hurwitz JL (2014): Respiratory tract epithelial cells express retinaldehyde dehydrogenase ALDH1A and enhance IgA production by stimulated B cells in the presence of vitamin A. PLoS One 9, e86554. https://doi.org/10.1371/ journal.pone.0086554

Sakuma S, Morimoto N, Nishida K, Murakami T, Egawa T, Endo R, Kataoka M, Yamashita S, Miyata K, Mohri K, Ochiai K, Hiwatari K, Koike S, Tobita E, Uto T, Baba M (2015): Cross-reactivity of immunoglobulin A secreted on the nasal mucosa in mice nasally inoculated with inactivated H1N1 influenza A viruses in the presence of D-octaarginine-linked polymers. Eur. J. Pharm. Biopharm. 92, 56-64. https://doi.org/10.1016/j.ejpb.2015.02.010

Stohr K (2002): Influenza-WHO cares. Lancet Infect. Dis. 2, 517. https://doi.org/10.1016/S1473-3099(02)00366-3

Surman SL, Jones BG, Sealy RE, Rudraraju R, Hurwitz JL (2014): Oral retinyl palmitate or retinoic acid corrects mucosal $\operatorname{IgA}$ responses toward an intranasal influenza virus vaccine in vitamin A deficient mice. Vaccine 32, 2521-2524. https://doi.org/10.1016/j.vaccine.2014.03.025

Tamura S, Iwasaki T, Thompson AH, Asanuma H, Chen Z, Suzuki Y, Aizawa C, Kurata T (1998): Antibody-forming cells in the nasal-associated lymphoid tissue during primary influenza virus infection. J. Gen. Virol. 79 ( Pt 2), 291-299. https://doi.org/10.1099/0022-1317-79-2-291 
Watanabe I, Hagiwara Y, Kadowaki SE, Yoshikawa T, Komase K, Aizawa C, Kiyono H, Takeda Y, McGhee JR, Chiba J, Sata T, Kurata T, Tamura S (2002): Characterization of protective immune responses induced by nasal influenza vaccine containing mutant cholera toxin as a safe adjuvant (CT112K). Vaccine 20, 3443-3455. https://doi. org/10.1016/S0264-410X(02)00351-1

Wiley JA, Tighe MP, Harmsen AG (2005): Upper respiratory tract resistance to influenza infection is not prevented by the absence of either nasal-associated lymphoid tissue or cervical lymph nodes. J. Immunol. 175, 3186-3196. https:// doi.org/10.4049/jimmunol.175.5.3186

Yang F, Wang D, Li Y, Sang L, Zhu J, Wang J, Wei B, Lu C, Sun X (2017): Th1/Th2 Balance and Th17/Treg-Mediated Immunity in relation to Murine Resistance to Dextran Sulfate-Induced Colitis. J. Immunol. Res. 2017, 7047201. https://doi.org/10.1155/2017/7047201
Yang P, Deng J, Li C, Zhang P, Xing L, Li Z, Wang W, Zhao Y, Yan Y, Gu H, Liu X, Zhao Z, Zhang S, Wang X, Jiang C (2012): Characterization of the 2009 pandemic A/Beijing/501/2009 H1N1 influenza strain in human airway epithelial cells and ferrets. PLoS One 7, e46184. https:// doi.org/10.1371/journal.pone.0046184

Yang P, Duan Y, Wang C, Xing L, Gao X, Tang C, Luo D, Zhao Z, Jia W, Peng D, Liu X, Wang X (2011): Immunogenicity and protective efficacy of a live attenuated vaccine against the 2009 pandemic A H1N1 in mice and ferrets. Vaccine 29, 698-705. https://doi.org/10.1016/j.vaccine.2010.11.026

Zeng H, Goldsmith C, Thawatsupha P, Chittaganpitch M, Waicharoen S, Zaki S, Tumpey TM, Katz JM (2007): Highly pathogenic avian influenza $\mathrm{H} 5 \mathrm{~N} 1$ viruses elicit an attenuated type i interferon response in polarized human bronchial epithelial cells. J. Virol. 81, 12439-12449. https://doi. org/10.1128/JVI.01134-07 